\title{
THE EQUALITY OF UNILATERAL DERIVATES
}

\author{
M. J. EVANS AND P. D. HUMKE
}

\begin{abstract}
C. J. Neugebauer has shown that if $f$ is a continuous function of bounded variation defined on the real line, then the set $E$ where the upper right derivate differs from the upper left derivate is of measure zero and first category. Here it is shown that this result is best possible; that is, given any measure zero first category set $K$, there is a continuous function of bounded variation for which $K \subseteq E$. It is also shown that if $f$ is monotone, then $E$ is $\sigma$-porous. This result can be used to provide an elementary proof of the fact that for an arbitrary function $f$ the left and right essential cluster sets are identical except at a $\sigma$-porous set of points, a result first proved by L. Zajičcek.
\end{abstract}

1. Introduction. Let $f$ be a real valued function defined on the real line $R$ and let

$$
E=\left\{x: f^{-}(x) \neq f^{+}(x) \text { or } f_{-}(x) \neq f_{+}(x)\right\},
$$

where $f^{-}(x)\left[f^{+}(x)\right]$ and $f_{-}(x)\left[f_{+}(x)\right]$ denote the left [right] upper and lower derivates of $f$ at $x$, respectively. C. J. Neugebauer [5] showed that if $f$ is of bounded variation and continuous then $E$ is of measure zero and first category. Using this result he provided an elementary proof of a result of $M$. Kulbacka [3] which states that for an arbitrary $f: R \rightarrow R$ the left and right essential cluster sets are equal except at those points in a set which is of measure zero and first category; in symbols, the set

$$
B=\left\{x: C_{e}^{-}(f, x) \neq C_{e}^{+}(f, x)\right\}
$$

is of measure zero and first category. L. Zajiček [6] has subsequently refined Kulbacka's result by showing that $B$ must be a $\sigma$-porous set.

The notion of a $\sigma$-porous set was introduced by E. P. Dolženko [2]. If $S$ is a set in $R$, the porosity of $S$ at the point $x$ in $R$ is defined to be

$$
\limsup _{r \downarrow 0} l(x, r, S) / r,
$$

where $l(x, r, S)$ denotes the length of the largest open interval contained in $(x-r, x+r) \cap(R \backslash S)$. The set $S$ is called porous if it has positive porosity at each of its points, and it is called $\sigma$-porous if it is a countable union of porous sets. Clearly, $\sigma$-porous sets must be of measure zero and first category. On the other hand, Zajíček [7] has constructed perfect measure zero sets which are not $\sigma$-porous.

A natural line of thought is to see if $B$ can be shown to be $\sigma$-porous by first obtaining an appropriate theorem concerning the derivates of some class of real functions and then applying Neugebauer's argument. This is accomplished in $\$ 2$ of

Received by the editors February 6, 1979 and, in revised form, September 23, 1979.

AMS (MOS) subject classifications (1970). Primary 26A24; Secondary 26A45, 26A48.

Key words and phrases. Derivates, bounded variation, monotone functions, $\sigma$-porosity. 
the present paper with the class being the collection of monotone functions. In $\$ 3$ we show that the class of continuous bounded variation functions is not small enough for our purpose in that given any measure zero first category set $K$, there is a continuous bounded variation function such that $K \subseteq E$.

2. Derivates of monotone functions. We begin by considering a theorem concerning the class of monotone functions and examine some consequences.

THEOREM 1. If $f: R \rightarrow R$ is monotone, then the set $E=\left\{x: f^{-}(x) \neq f^{+}(x)\right.$ or $\left.f_{-}(x) \neq f_{+}(x)\right\}$ is $\sigma$-porous.

Proof. Suppose that $f$ is nondecreasing. We shall show that the set $A=\{x$ : $\left.f^{-}(x)<f^{+}(x)\right\}$ is $\sigma$-porous.

The remaining cases are all handled in a similar fashion.

For every pair $(r, s)$ of rational numbers satisfying $0<r<s$, let

$$
A_{r s}=\left\{x: f^{-}(x)<r<s<f^{+}(x)\right\} .
$$

It then suffices to show that each $A_{r s}$ is $\sigma$-porous. For each natural number $n$, let

$$
A_{r s n}=\left\{x: x \in A_{r s} \text { and } \frac{f(y)-f(x)}{y-x}<r \text { for } x-1 / n<y<x\right\} .
$$

We shall show that $A_{r s n}$ is a porous set. Indeed, $A_{r s n}$ will have porosity greater than or equal to $1-r / s$ at each of its points.

To see this, let $x \in A_{r s n}$. Then there is a sequence of numbers $x_{k}$ converging to $x$ from the right such that

$$
\frac{f\left(x_{k}\right)-f(x)}{x_{k}-x}>s .
$$

Take $k$ so large that $x_{k}-x<1 / n$, let $w_{k}=x+s\left(x_{k}-x\right) / r$, and let $I_{k}=$ $\left[x_{k}, w_{k}\right]$. Then for $y \in I_{k}$ we have

$$
\frac{x_{k}-x}{y-x}>\frac{r}{s}
$$

and using this along with the monotonicity of $f$, it follows that

$$
\frac{f(y)-f(x)}{y-x}>\frac{f\left(x_{k}\right)-f(x)}{x_{k}-x} \cdot \frac{x_{k}-x}{y-x}>r .
$$

Consequently, $y \notin A_{r s n}$. Furthermore, for each $k$ we have

$$
\frac{\left|I_{k}\right|}{w_{k}-x}=1-\frac{r}{s}
$$

and hence $A_{r s n}$ has porosity at least $1-r / s$ at $x$.

Using this result and the argument employed by Neugebauer [5], we obtain the following

COROLLARY 1. For an arbitrary function $f: R \rightarrow R$ the set

$$
B=\left\{x: C_{e}^{-}(f, x) \neq C_{e}^{+}(f, x)\right\}
$$

is o-porous. 
Next we present an application of Theorem 1 to other types of derivates. Let $f^{s}(x)$ denote the upper symmetric derivate of $f$ at $x$, that is,

$$
f^{s}(x)=\lim _{h \rightarrow 0^{+}} \sup \frac{f(x+h)-f(x-h)}{2 h} .
$$

In [1] it was shown that if $f: R \rightarrow R$ is continuous at a dense set of points, then $f^{s}(x)=\max \left\{f^{+}(x), f^{-}(x)\right\}$ except at a $\sigma$-porous set of points.

Further, let $f_{a p}^{+}(x), f_{a p}^{-}(x)$, and $f_{\text {ap }}^{s}(x)$ denote the upper approximate right, left, and symmetric derivates of $f$ at $x$, respectively. L. Miší [4] has shown that if $f$ is monotone, then $f_{a p}^{+}(x)=f^{+}(x)$ and $f_{a p}^{-}(x)=f^{-}(x)$ pointwise. A very similar proof shows that $f_{a p}^{s}(x)=f^{s}(x)$ pointwise for a monotone function.

Combining Theorem 1 and the information in the previous two paragraphs, we obtain

COROLlaRY 2. If $f: R \rightarrow R$ is monotone, then except at those points $x$ in a o-porous set we have

$$
f^{+}(x)=f^{-}(x)=f^{s}(x)=f_{a p}^{s}(x)=f_{a p}^{-}(x)=f_{a p}^{+}(x) .
$$

3. The inequality of unilateral derivates. For purposes of clarity we will divide this section into two parts. In the first part we prove that given any perfect set $K$ of measure zero, there is a continuous bounded variation function such that $K \subseteq E$ (Theorem 2). The remainder of this section is then devoted to generalizing Theorem 2 to first category sets $K$ of measure zero.

As the actual construction used in Theorem 2 is somewhat complicated, we first describe a function defining process which will be used in an inductive manner in the final construction. Let $I=[u, v]$ be a closed interval and let $K$ be a perfect set of measure zero contained in $I$ such that both $u$ and $v$ are in $K$. In addition, let $\varepsilon>0$ be specified. The function we wish to define will be zero except at enumerably many contiguous intervals of very small cumulative measure; the union of these intervals will be denoted by $B$. Another finite set of contiguous intervals of large cumulative measure is then specified; the union of these intervals is denoted by $A$. In addition, $A$ and $B$ are chosen so that the remainder of $I$ consists of enumerably many closed intervals whose union is denoted by $C$. Specifically, let $A=\cup{ }_{k=1}^{N}\left(a_{k}, a_{k}^{\prime}\right)$ where each interval $\left(a_{k}, a_{k}^{\prime}\right)$ is contiguous to $K, a_{k}^{\prime}<a_{k+1}, k=$ $1,2, \ldots, N-1$, and $\Sigma\left(a_{k}^{\prime}-a_{k}\right)>v-u-\varepsilon / 4$. For notational convenience, let $u=a_{0}^{\prime}$ and $v=a_{N+1}$. Now, in each interval $\left[a_{k}^{\prime}, a_{k+1}\right], k=0,1, \ldots, N$, there is a sequence of intervals $\left\{\left(b_{k i}, b_{k i}^{\prime}\right): i=1,2, \ldots\right\}$ each contiguous to $K$ such that $b_{k i}^{\prime}<b_{k(i+1)}$, and $\lim _{i \rightarrow \infty} b_{k i}=a_{k+1}$. For notational convenience we let $b_{k 0}^{\prime}=a_{k}^{\prime}$, and let

$$
B=\bigcup_{k=0}^{N} \bigcup_{i=1}^{\infty}\left(b_{k i}, b_{k i}^{\prime}\right) \text { and } C=I-A \cup B .
$$

Define $F(I, K, \varepsilon, A, B)=F(I, K, \varepsilon)$ as follows: $F$ is to be zero except at the intervals $\left[b_{k i}, b_{k i}^{\prime}\right], k=0,1, \ldots, N, i=1,2, \ldots$; on each interval $\left[b_{k i}, b_{k i}^{\prime}\right]$ define $F$ to be a quadratic function with zeros at both $b_{k i}$ and $b_{k i}^{\prime}$, and a maximum of 
$\left(b_{k i}+b_{k i}^{\prime}-2 b_{k(i-1)}^{\prime}\right) / 2$. First note that $F$ is continuous, $F(K)=\{0\}, F$ is zero outside of $I$, and the variation of $F$ is less than $\varepsilon / 2$. Now, if $x \in K$ and $x$ is neither the left endpoint of an interval in $A$ nor the maximum of $K$, then there is a $y \in I$ such that $[f(y)-f(x)] /[y-x]>1$. This " $y$ " can be chosen to be the midpoint of the " $B$ interval" immediately to the right of $x$.

Generalizing slightly, let $I$ be the union of a finite or denumerable set of mutually exclusive closed intervals, $I=\cup I_{n}$. Suppose $K$ is a set such that $K \cap I_{n}$ is a perfect set of measure zero and $K$ contains both endpoints of $I_{n}$ for every $n$. Suppose further that $\varepsilon>0$ is specified. Define $F\left(I_{n}, K \cap I_{n}, \varepsilon / 2^{n-1}, A_{n}, B_{n}\right) \equiv$ $F\left(I_{n}, K \cap I_{n}, \varepsilon / 2^{n-1}\right)$ as above and let

$$
F(I, K, \varepsilon)(x)= \begin{cases}F\left(I_{n}, K \cap I_{n}, \varepsilon / 2^{n-1}\right)(x) & \text { if } x \in I_{n}, \\ 0 & \text { if } x \notin I .\end{cases}
$$

In this case the variation of $F$ is less than $\sum \varepsilon / 2^{n}<\varepsilon$. The bother of identifying the sets $A, B$, and $C$ for such functions is necessitated by the induction argument used in the proof below.

THEOREM 2. If $K \subset(a, b)$ is a perfect set of measure zero and $\varepsilon>0$, then there is a continuous function of bounded variation, $f: R \rightarrow[0,+\infty)$ such that

(1) $f(x)=0$ if either $x \in K$ or $x \notin(a, b)$,

(2) $f^{+}(x)>1$ and $f^{-}(x)<0$ for every $x \in K$,

(3) the total variation of $f, V(f)$, is less than $\varepsilon$, and

(4) $f$ is differentiable at every point not in $K$.

Proof. Let $u=\min (K), v=\max (K)$, and $I_{1}=[u, v]$. Let $f_{1} \equiv$ $F\left(I_{1}, K, \varepsilon / 4, A_{1}, B_{1}\right)$. Suppose now that $f_{n}$ has been defined and is of the form $F\left(I_{n}, K, \varepsilon / 2^{n+1}, A_{n}, B_{n}\right)$ for the union $I_{n}$ of some family of mutually exclusive closed intervals. Let $I_{n+1}=C_{n}\left(C_{n}=I_{n}-A_{n} \cup B_{n}\right)$ and define $f_{n+1} \equiv$ $F\left(I_{n+1}, K, \varepsilon / 2^{n+2}, A_{n+1}, B_{n+1}\right)$. This completes the induction and we define $f^{*}=$ $\sum f_{n}$.

It is evident that $f^{*}$ is continuous. And as $f^{*}(K)=0$ and $f^{*}(x)>0$ for each $x,\left(f^{*}\right)^{-}(x)<0$ for every $x \in K$. Also, as the variation of $f_{n}$ is less than $\varepsilon / 2^{n+1}, f^{*}$ is of bounded variation and the variation of $f^{*}$ is less than $\varepsilon / 2$. Let $A=\cup_{n=1}^{\infty} A_{n}$ and $B=\cup_{n=1}^{\infty} B_{n}$ and suppose $x \in K$ where $x \neq v$ and $x$ is not the left endpoint of an interval from either $A$ or $B$. Such an $x$ is the intersection of a nested set of intervals $C_{n}(x) \subseteq C_{n}$ and $x$ is not the right endpoint of any of the $C_{n}(x)$ intervals. Consequently, there is a point $y_{n} \in C_{n}(x)$ such that $\left[f^{*}\left(y_{n}\right)-f^{*}(x)\right] /\left[y_{n}-x\right]>1$. It follows that for such $x,\left(f^{*}\right)^{+}(x)>1$. If $x$ is a left endpoint of an interval in $B$, then since $F_{+}^{\prime}\left(b_{k i}\right)>1$ for each $k$ and $i$, there is an $n$ such that $\left(f_{n}\right)_{+}^{\prime}(x)>1$, whence $\left(f^{*}\right)^{+}(x)>1$. We must alter $f^{*}$ slightly in order that the upper right derivate exceeds 1 for $v$ and for left endpoints of intervals in $A$, and the remainder of the proof is devoted to this task. First note that $A$ is the union of those intervals contiguous to $K$ which have been mapped to zero by $f^{*}$. Enumerate those intervals as $\left\{\left(a_{n}, b_{n}\right): n=1,2, \ldots\right\}$, and for notational convenience, let $v=a_{0}$ and $b=b_{0}$. For each interval, $\left(a_{n}, b_{n}\right) n=0,1,2, \ldots$, let $c_{n} \in\left(a_{n}, b_{n}\right)$ such that $c_{n}-a_{n}<$ $\varepsilon / 2^{n+2}$. Define a function $g: R \rightarrow[0,+\infty)$ to be zero except on the intervals 
$\left[a_{n}, c_{n}\right] n=0,1,2, \ldots$; on each interval $\left[a_{n}, c_{n}\right]$ define $g$ to be a cubic function with a simple zero at $a_{n}$, a double zero at $c_{n}$, and a maximum of $\left(c_{n}-a_{n}\right) / 2$. This function $g$ is complementary to $f^{*}$ in the sense that $g^{-1}(0,+\infty) \cap f^{*-1}(0,+\infty)=$ $\varnothing$. The desired function is then $f=f^{*}+g$. This completes the proof of Theorem 2 .

THEOREM 3. Let $K$ be a set which is of measure zero, and of the first Baire category. Then there is a continuous function $f$ of bounded variation such that $\left|f^{+}(x)-f^{-}(x)\right|>1$ for every $x \in K$.

Proof. As $K$ is of measure zero and the first Baire category, a standard set theoretic argument shows that there is a set of mutually exclusive perfect sets of measure zero, $\left\{K_{n}: n=1,2, \ldots\right\}$ such that $K \subseteq \cup_{n=1}^{\infty} K_{n}$. Let $f_{1}$ be a continuous nonnegative bounded variation function such that:

(1) $f_{1}\left(K_{1}\right)=\{0\}$,

(2) $f_{1}^{+}(x)>1$ and $f_{1}^{-}(x)<0$ for every $x \in K_{1}$,

(3) $V\left(f_{1}\right)<\frac{1}{2}$, and

(4) $f_{1}$ is differentiable at every point not in $K_{1}$.

Denote the set of intervals contiguous to $K_{1}$ by $\left\{\left(a_{i}, b_{i}\right): i=1,2, \ldots\right\}$. On each interval $\left(a_{i}, b_{i}\right)$ define $f_{2}$ as follows:

(i) If $K_{2} \cap\left(a_{i}, b_{i}\right)=\varnothing$, let $f_{2}\left(a_{i}, b_{i}\right)=\{0\}$.

(ii) If $K_{2} \cap\left(a_{i}, b_{i}\right) \neq \varnothing$, then $K_{2} \cap\left(a_{i}, b_{i}\right)$ is perfect and we define $f_{2}$ on $\left(a_{i}, b_{i}\right)$ using Theorem 2 with $\varepsilon=1 / 2^{i+2}$.

Finally, we define $f_{2}\left(K_{1}\right)=\{0\}$. Note that $V\left(f_{2}\right)<\frac{1}{4}$ so that the variation of $g=f_{1}+f_{2}$ is less than $\frac{3}{4}$. Further, if $x \in K_{1}, g^{+}(x)>1$ and $g^{-}(x)<0$ and if $x \in K_{2}, g^{+}(x)>1+s$ and $g^{-}(x)<s$ where $s$ is the derivative of $f_{1}$ at $x$.

We continue inductively to obtain for each $n$ a function $f_{n}$ of variation less than $1 / 2^{n}$. Let $f=\sum_{n=1}^{\infty} f_{n}$. If $x \in \cup_{n=1}^{\infty} K_{n}$, then there is a unique $N=N(x)$ such that $x \in K_{N}$. Now for $n>N, f_{n}(x)=0$ and the construction entails that

$$
\left(f_{N}+f_{N+1}+\ldots\right)^{+}(x)>1, \quad\left(f_{N}+f_{N+1}+\ldots\right)^{-}(x)<0,
$$

and each of the functions $f_{1}, f_{2}, \ldots, f_{N-1}$ has a derivative at $x$. It follows that $\left|f^{+}(x)-f^{-}(x)\right|>1$ for each such $x$, and as $f$ is evidently continuous and of variation less than one, the proof is complete.

\section{REFERENCES}

1. C. L. Belna, M. J. Evans and P. D. Humke, Symmetric and ordinary differentiation, Proc. Amer. Math. Soc. 72 (1978), 261-267.

2. E. P. Dolženko, Boundary properties of arbitrary functions, Math. USSR-Izv. 1 (1967), 1-12.

3. M. Kulbacka, Sur l'ensemble des points de l'asymetrie approximative, Acta Sci. Math. (Szeged) 21 (1960), 90-95.

4. L. Mišik, Über approximative derivierte Zahlen monotoner Funktionen, Czechoslovak Math. J. 26 (101) (1976), 579-583.

5. C. J. Neugebauer, $A$ theorem on derivates, Acta Sci. Math. (Szeged) 23 (1962), 79-81.

6. L. Zajicek, On cluster sets of arbitrary functions, Fund. Math. 83 (1974), 197-217.

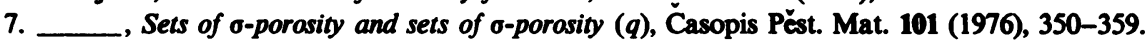

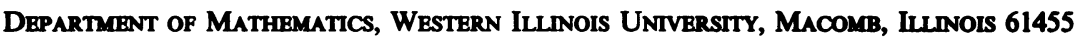

